\title{
Light propagation and the average expansion rate in near-FRW universes
}

\section{Räsänen, Syksy}

2012

Räsänen , S 2012 , ' Light propagation and the average expansion rate in near-FRW universes ' , Physical Review D : Particles, Fields, Gravitation and Cosmology , vol. 85 , no. 8 , pp. 083528 . https://doi.org/10.1103/PhysRevD.85.083528

http://hdl.handle.net/10138/310414

https://doi.org/10.1103/PhysRevD.85.083528

acceptedVersion

Downloaded from Helda, University of Helsinki institutional repository.

This is an electronic reprint of the original article.

This reprint may differ from the original in pagination and typographic detail.

Please cite the original version. 


\title{
Light propagation and the average expansion rate in near-FRW universes
}

\author{
Syksy Räsänen \\ University of Helsinki, Department of Physics \\ and Helsinki Institute of Physics \\ P.O. Box 64, FIN-00014 University of Helsinki, Finland \\ syksy dot rasanen at iki dot fi
}

\begin{abstract}
We consider universes that are close to Friedmann-Robertson-Walker in the sense that metric perturbations, their time derivatives and first spatial derivatives are small, but second spatial derivatives are not constrained. We show that if we in addition assume that the observer four-velocity is close to its background value and close to the four-velocity which defines the hypersurface of averaging, the redshift and the average expansion rate remain close to the FRW case. However, this is not true for the angular diameter distance. The four-velocity assumption implies certain conditions on second derivatives of the metric and/or the matter content.
\end{abstract}




\section{Contents}

1. Introduction 1

2. The spacetime geometry 3

2.1 Kinematics and the equation of motion 3

2.2 The near-FRW assumption

3. Light propagation

3.1 The redshift 7

3.2 The distance 8

4. The expansion rate 9

4.1 The local expansion rate 9

4.2 The average expansion rate 10

4.3 The background expansion rate 11

5. Discussion 13

5.1 The Buchert equations 13

5.2 The redshift and the average expansion rate 14

5.3 Relation to Newtonian gravity 15

5.4 Local and global backgrounds 17

6. Conclusion 19

\section{Introduction}

The matter-dominated homogeneous and isotropic cosmological model based on general relativity disagrees with observations at late times. The observed angular diameter distance to the last scattering surface at redshift 1090 is a factor of 1.4-1.9 longer (keeping the Hubble constant fixed) [1,2], and the expansion rate is larger by a factor of $1.2-1.7$ (keeping the age of the universe fixed, i.e. $H_{0} t_{0} \approx 0.8 \ldots 1.1$ ) $[2,3]$ or by a factor of 1.6-2.2 (keeping the matter density fixed, i.e. $\Omega_{m 0} \approx 0.2 \ldots 0.4$ ) $[1,2,4]$. The usual remedy is to either include exotic matter with negative pressure or modify the law of gravity. However, homogeneous and isotropic models do not include the effect of non-linear structures on the expansion of the universe and on light propagation, 
and the factor two failure of the predictions of the matter-dominated model could be related to this shortcoming [5-9].

The effect of inhomogeneities on the average expansion rate is called backreaction [10-12]; see [13-16] for reviews. It has been shown in toy models that non-linearities can lead to faster expansion, even acceleration, for dust matter [14,17-21]. In a semirealistic model, the observed timescale and the order of magnitude of the change in the expansion rate emerge from the physics of structure formation [22,23], but there is no fully realistic calculation yet. If backreaction is significant (and the universe is statistically homogeneous and isotropic with a homogeneity scale smaller than the horizon), this has to be due to non-Newtonian aspects of gravity $[11,12,14,15,22$, 24-28] which are related to the difference between Newtonian gravity and the weak field, small velocity limit of general relativity [29-36].

The magnitude of the effect in the real universe remains an open question. It has been argued that backreaction is small because the universe is close to a homogeneous and isotropic Friedmann-Robertson-Walker (FRW) model at all times. However, we should be specific ab out what is meant with the statement that the universe is close to FRW. Smallness of metric perturbations does not preclude large deviations in the Riemann tensor, because the latter involves second derivatives of the metric, and the variation of a function may be rapid (with regard to some relevant scale) even though its amplitude is small. This is the case in cosmology when density perturbations enter the non-linear regime.

The argument involves two separate questions. First, can the universe can be described with a metric which is perturbatively close to the FRW metric even after density perturbations are non-linear? Second, does smallness of metric perturbations imply that the average expansion rate, the redshift and the angular diameter distance remain close to their unperturbed values?

The second question has been studied in many papers with regard to the average expansion rate [7,9,24,37-43] (see [24] for further discussion and references). However, almost all studies have been restricted to first or second order perturbation theory and/or have had other shortcomings [24] $]^{1}$. A notable exception is [44], which considers a new perturbative formalism adapted to the cosmological situation where ordinary perturbation theory is not applicable; see section 5.4. There is also a large literature on non-linear effects in light propagation, starting with a paper by Zel'dovich in 1964 [45] (see [22] for further discussion and references). However, the question phrased above has rarely been the focus of light propagation studies, and it has not received a definitive answer.

We assume that metric perturbations remain small, and concentrate on the second issue. The expansion rate and the distance both involve second derivatives of the metric, like the density perturbation. The question is not whether it is possible

\footnotetext{
${ }^{1}$ In [43], the average is taken over the background FRW volume as opposed to the physical volume, so the central issue of the non-commutativity of time derivatives and averaging is missing.
} 
to have large local deviations: the variation of the local expansion rate between different regions is of order unity in the real universe. The issue is whether it follows from the smallness of metric perturbations that the distribution of the expansion rate is such that the fluctuations cancel in the average, and whether corrections to the distance and redshift are correspondingly small.

We show that if the spacetime is close to FRW in the sense that metric perturbations, their time derivatives and first spatial derivatives are small and if the observer four-velocity is close to its background value and close to the four-velocity which defines the hypersurface of averaging, the redshift and the average expansion rate remain close to the FRW case. Such a result does not hold for the angular diameter distance.

In section 2 we set up the formalism and state our assumptions and in section 3 we show that the change in the redshift is small and explain why this is not the case for the angular diameter distance. In section 1 we consider the average expansion rate. In section 5 we discuss our results, in particular the relation to Newtonian gravity, and in section 6 we summarise the situation.

\section{The spacetime geometry}

\subsection{Kinematics and the equation of motion}

Two frames. We mostly follow the notation of [46]; for reviews of the covariant formalism, see [29, 47-49]. We denote the four-velocity of the observers by $u^{\alpha}$. We consider a spacelike hypersurface $\mathcal{N}$ and denote the unit vector orthogonal to $\mathcal{N}$ by $n^{\alpha}$. We will take averages on this hypersurface; at this stage, $\mathcal{N}$ is completely general. Both vectors are normalised to unity, $u_{\alpha} u^{\alpha}=n_{\alpha} n^{\alpha}=-1$. The tensors which project on the hypersurface orthogonal to $n^{\alpha}$ and the rest space orthogonal to $u^{\alpha}$ are, respectively,

$$
\begin{aligned}
& h_{\alpha \beta} \equiv g_{\alpha \beta}+n_{\alpha} n_{\beta} \\
& h_{\alpha \beta}^{(u)} \equiv g_{\alpha \beta}+u_{\alpha} u_{\beta},
\end{aligned}
$$

and we denote by $\hat{\nabla}_{\alpha}$ the spatial covariant derivative which is completely projected on $\mathcal{N}$, e.g. $\hat{\nabla}_{\beta} f_{\alpha}=h_{\beta}^{\delta} h_{\alpha}^{\gamma} \nabla_{\delta} f_{\gamma}$. Without loss of generality, we write

$$
u^{\alpha}=\gamma\left(n^{\alpha}+v^{\alpha}\right)
$$

where $v_{\alpha} n^{\alpha}=0$ and $\gamma=-n_{\alpha} u^{\alpha}=\left(1-v^{2}\right)^{-1 / 2}$ with $v^{2} \equiv v_{\alpha} v^{\alpha}$.

It is useful to decompose the gradient of $n_{\alpha}$ as

$$
\nabla_{\beta} n_{\alpha}=\frac{1}{3} h_{\alpha \beta} \theta+\sigma_{\alpha \beta}-A_{\alpha} n_{\beta},
$$


where $\theta \equiv \nabla_{\alpha} n^{\alpha}$ is the volume expansion rate, $\sigma_{\alpha \beta} \equiv \hat{\nabla}_{(\beta} n_{\alpha)}-\frac{1}{3} h_{\alpha \beta} \theta$ is the shear tensor and $A_{\alpha} \equiv n^{\beta} \nabla_{\beta} n_{\alpha}$ is the acceleration vector. The analogous decomposition of the gradient of $u_{\alpha}$ is

$$
\nabla_{\beta} u_{\alpha}=\frac{1}{3} h_{\alpha \beta}^{(u)} \theta^{(u)}+\sigma_{\alpha \beta}^{(u)}+\omega_{\alpha \beta}^{(u)}-A_{\alpha}^{(u)} u_{\beta}
$$

where $\omega_{\alpha \beta}^{(u)} \equiv \nabla_{[\beta} u_{\alpha]}+A_{[\alpha}^{(u)} u_{\beta]}$ is the vorticity tensor and the other quantities are defined in the same manner as those in (2.3).

We assume that the relation between the geometry and the matter content is given by the Einstein equation (we use units in which $8 \pi G_{\mathrm{N}}=1, G_{\mathrm{N}}$ being Newton's constant),

$$
G_{\alpha \beta}=T_{\alpha \beta}=\rho u_{\alpha} u_{\beta}+p h_{\alpha \beta}^{(u)}+2 q_{(\alpha} u_{\beta)}+\pi_{\alpha \beta}
$$

where we have without loss of generality decomposed the energy-momentum tensor $T_{\alpha \beta}$ with respect to $u^{\alpha}$. Here $\rho \equiv u^{\alpha} u^{\beta} T_{\alpha \beta}$ is the energy density, $p \equiv \frac{1}{3} h^{\alpha \beta} T_{\alpha \beta}$ is the pressure, $q_{\alpha} \equiv-h_{\alpha}^{\beta} u^{\gamma} T_{\beta \gamma}$ is the energy flux and $\pi_{\alpha \beta} \equiv h_{\alpha}^{\gamma} h_{\beta}^{\delta} T_{\gamma \delta}-\frac{1}{3} h_{\alpha \beta} h^{\gamma \delta} T_{\gamma \delta}$ is the anisotropic stress.

\subsection{The near-FRW assumption}

The metric. We write the metric as

$$
\mathrm{d} s^{2}=-(1+2 \Phi) \mathrm{d} \bar{t}^{2}+2 \alpha_{i} \mathrm{~d} \bar{t} \mathrm{~d} x^{i}+\left([1-2 \Psi] f_{i j}+\chi_{i j}\right) a(\bar{t})^{2} \mathrm{~d} x^{i} \mathrm{~d} x^{j}
$$

where $f_{i j} \equiv\left(1+K \delta_{k l} x^{k} x^{l} / 4\right)^{-2} \delta_{i j} \equiv f \delta_{i j}$ is the metric of a three-dimensional homogeneous and isotropic space with constant curvature $6 K / a^{2}$. The scale factor is normalised to unity today, $a\left(\bar{t}_{0}\right)=1$. We have $\delta^{i j} \chi_{i j}=0$. We define $H \equiv \dot{a} / a$, where dot means derivative with respect to the coordinate time $\bar{t}$. This form of the metric is completely general. We refer to the spacetime obtained when $\Phi=\Psi=0, \alpha_{i}=0, \chi_{i j}=0$ as the background, and refer to these functions as perturbations. We choose the Poisson gauge, which is defined by $\delta^{i j} \alpha_{i \mid j}=0, \delta^{j k} \chi_{i j \mid k}=0$, where / indicates covariant derivative with respect to $f_{i j}$.

We want the spacetime to be close to FRW and the coordinate system to be close to the coordinates where the background looks homogeneous and isotropic, so we assume that the metric perturbations are small. For the scalar functions $\Phi$ and $\Psi$, we can simply demand that their values are small everywhere. The magnitude of $\alpha_{i}$ and $\chi_{i j}$ depends on the coordinate system, so we have to be a bit more careful. If the background space is negatively curved, $f$ diverges at $r=2 / \sqrt{-K}$, and $f$ approaches zero as $r$ goes to infinity for either negative or positive spatial curvature. Correspondingly, if a field $A^{i}$ has finite norm with regard to the background space, $\bar{g}_{i j} A^{i} A^{j}=a^{2} f \delta_{i j} A^{i} A^{j}$ (where $\bar{g}_{i j}$ is the spatial background metric), the components $A^{i}$ will vanish or diverge as $f$ diverges or vanishes, respectively. We define the 
background-normalised spatial components of any field as $A^{\hat{i}} \equiv a \sqrt{f} A^{i}$ and $A_{\hat{i}} \equiv$ $(a \sqrt{f})^{-1} A_{i}$ (and correspondingly for fields with more than one spatial index) to avoid this coordinate divergence. The requirement that the perturbations are small can now be stated as $\epsilon(x) \equiv \max \left(|\Phi|,|\Psi|,\left|\alpha_{\hat{i}}\right|,\left|\chi_{\hat{i} \hat{j}}\right|\right) \ll 1$. For $\alpha_{i}$ and $\chi_{i j}$ we can equivalently say $\delta^{i j} \alpha_{\hat{i}} \alpha_{\hat{j}}=\bar{g}^{i j} \alpha_{i} \alpha_{j} \lesssim \epsilon^{2}, \delta^{i k} \delta^{j l} \chi_{\hat{i} \hat{j}} \chi_{\hat{k} \hat{l}}=\bar{g}^{i k} \bar{g}^{j l} \chi_{i j} \chi_{k l} \lesssim \epsilon^{2}$. In summary, we assume that the spacetime metric is everywhere perturbatively near the same global background. We further assume that the background spatial curvature is not significantly larger than the background expansion rate, $|K| / a^{2} \lesssim H^{2}$, i.e. we do not consider near-static spacetimes.

We are interested in modes whose wavelengths are not long, that is to say modes for which first spatial derivatives are large compared to (or of the same order as) the perturbations, but still smaller than unity, given $a|H|$ as the comparison scale, $1 \gg\left|\tilde{\partial}_{\hat{i}} \epsilon\right| \gtrsim \epsilon$, where we have defined $\tilde{\partial}_{\hat{i}} \equiv(a \sqrt{f})^{-1} \partial_{i} /|H|{ }^{2}$ We also assume that time evolution is slow compared to spatial changes, more precisely that $|\dot{\epsilon}| \lesssim|H| \epsilon$, i.e. time derivatives are at most of the same order of magnitude as the background time scale. We make no assumptions about second derivatives of the metric perturbations, they can be comparable to the background quantities or larger.

The Einstein tensor. The components of the Einstein tensor for the near-FRW metric (2.6) are

$$
\begin{aligned}
G_{00} \simeq & 3 H^{2}+3 \frac{K}{a^{2}}+2 \Psi_{\mid k}^{\mid k}+\mathcal{O}\left(\epsilon \partial^{2} \epsilon, \partial \epsilon \partial \epsilon, \bar{\Gamma} \epsilon \partial \epsilon, \partial \bar{\Gamma} \epsilon, \bar{\Gamma}^{2} \epsilon, \bar{\Gamma} H \epsilon, H^{2} \epsilon\right) \\
G_{0 \hat{i}} \simeq & -\frac{1}{2} \alpha_{\hat{i} \mid k}^{\mid k}+\frac{K}{2 f^{\frac{1}{2}} a^{2}} x^{k} \partial_{k} \alpha_{\hat{i}}+2 \partial_{\hat{i}}(\dot{\Psi}+H \Phi)+\mathcal{O}\left(\epsilon \partial^{2} \epsilon, \partial \bar{\Gamma} \epsilon, \bar{\Gamma}^{2} \epsilon, \bar{\Gamma} H \epsilon, H^{2} \epsilon\right)\left(2 .{ }^{2}\right) \\
G_{\hat{i} \hat{j}} \simeq & -\left(2 \frac{\ddot{a}}{a}+H^{2}+\frac{K}{a^{2}}\right) \delta_{i j}+(\Psi-\Phi)^{\mid i}{ }_{\mid j}-(\Psi-\Phi)^{\mid k}{ }_{\mid k} \delta_{i j}-\partial_{(\hat{i}} \dot{\alpha}_{\hat{j})}-2 H \partial_{(\hat{i}} \alpha_{\hat{j})} \\
& -\frac{1}{2} a^{2} \chi_{\hat{i}_{\hat{i}}|k| k}+\frac{K}{f^{\frac{1}{2}}} x^{k} \partial_{k} \chi_{\hat{i} \hat{j}}+\mathcal{O}\left(\epsilon \partial^{2} \epsilon, \partial \epsilon \partial \epsilon, \bar{\Gamma} \epsilon \partial \epsilon, \partial \bar{\Gamma} \epsilon, \bar{\Gamma}^{2} \epsilon, \bar{\Gamma} H \epsilon, H^{2} \epsilon\right)
\end{aligned}
$$

where $\simeq$ indicates dropping subleading terms in metric perturbations and their derivatives; in the remainder terms we have not kept track of the indices. We use the symbol $\bar{\Gamma}$ to refer to the background spatial Christoffel symbols; $\partial$ indicates $\partial_{\hat{i}}$ and $\partial^{2}$ indicates a combination of two spatial derivatives (likewise for $\tilde{\partial}$ and $\tilde{\partial}^{2}$ ). To simplify the bookkeeping, we take in what follows $\bar{\Gamma} \lesssim|H|$, in line with the assumption that we do not consider near-static universes.

Because the Einstein equation is second order, there are at most two derivatives acting on a metric perturbation, so the structure remains close to linear theory.

\footnotetext{
${ }^{2}$ If the background expansion is expanding and decelerating, this condition becomes stronger over time, since $1 /(a|H|)$ increases. Conversely, in an accelerating expanding background the condition becomes weaker. For a collapsing background the situation is reversed. This is assuming that the time-dependence of $\epsilon$ does not overcome that of $a|H|$.
} 
Note that this not an expansion in powers of the metric perturbation: in that case perturbations and their derivatives would be considered to be of the same order [50]. When derivatives are large, this is inconsistent, as the first and second derivatives of the metric perturbations are effectively new expansion parameters [24] (see also [44]).

The four-velocity. Observables such as the redshift, the angular diameter distance and the local expansion rate depend on the observer four-velocity $u^{\alpha}$. According to observations, deviations of galaxies from the mean flow are small over large scales. These local deviations often go by the name peculiar velocities. In linear theory and in the Poisson gauge, it is simple to identify $u^{i}$ as the physical velocity around the mean flow. However, defining the peculiar velocity in a more general context and translating the observational constraint into a well-defined mathematical statement is not straightforward $[49,51]$.

The difference between the actual value of $u^{\alpha}$ and its background value is gaugedependent, so the physical meaning of it being small is not obvious. (Requiring metric perturbations to remain small is open to the same criticism.) For example, it is always possible to adopt the comoving gauge where $u^{i}=0$, though then metric perturbations become large at the same time as density perturbations. On the other hand, $\left|u^{\hat{i}}\right| \sim 1$ does not necessarily contradict any observations, any more than metric perturbations of order unity do. The physical peculiar velocity would need to be defined with respect to a physically defined velocity field describing the mean flow. We will simply look at the difference from the background in the Poisson gauge, like we do with the metric perturbations. For the background we have $\bar{u}^{\alpha}=\delta^{\alpha 0}$, so we are interested in whether the conditions $\left|u^{0}-1\right|,\left|u^{\hat{i}}\right| \ll 1$ hold. Given the normalisation $g_{\alpha \beta} u^{\alpha} u^{\beta}=-1$ and the smallness of metric perturbations, the first condition follows from the second, so we only need to check whether $\left|u^{\hat{i}}\right| \ll 1$. From the $0 i$ component of (2.5), we have

$$
u_{\hat{i}}=\frac{G_{0 \hat{i}}-\alpha_{\hat{i}} p-u_{0} q_{\hat{i}}-\pi_{0 \hat{i}}}{(\rho+p) u_{0}+q_{0}},
$$

The relation (2.10) shows what is required in terms of metric perturbations and the matter content to keep $u_{\hat{i}}$ small. According to (2.8) the leading contribution to $G_{0 \hat{i}}$ is $\alpha_{\hat{i} \mid k}^{\mid k} \simeq(a \sqrt{f})^{-3 / 2} \nabla^{2} \alpha_{i}$, so smallness of metric perturbations and their first derivatives is not enough to guarantee that $u_{\hat{i}}$ would remain small ${ }^{3}$. We could make the additional assumption $\left|\alpha_{\hat{i} \mid k}^{\mid k} / H^{2}\right| \lesssim\left|\tilde{\partial}_{\epsilon}\right|$ for the metric, and assume for the matter content that $\left|q_{\hat{i}} /(\rho+p)\right| \lesssim|\tilde{\partial} \epsilon|,\left|\pi_{0 \hat{i}} /(\rho+p)\right| \lesssim|\tilde{\partial} \epsilon|$, and that there is no negative pressure so large that we would have $|\rho+p| \ll \rho$. (Note that the magnitude of $\pi_{\hat{i} \hat{j}}$ is unconstrained.) Under these conditions, (2.10) gives $\left|u_{\hat{i}}\right| \ll 1$. These conditions are sufficient, but not necessary, as there can be cancellations among the different terms

\footnotetext{
${ }^{3}$ It is important that time derivatives of metric perturbations remain small, otherwise $u_{\hat{\imath}}$ is in general large, as $G_{0 \hat{i}}$ always involves second derivatives of the metric.
} 
in (2.10). We will therefore simply assume that $u_{\hat{i}} \sim \mathcal{O}(\tilde{\partial} \epsilon)$ without specifying which of these conditions hold.

If the observer motion is geodesic, $A_{\alpha}^{(u)}=0$, it follows from the geodesic equation that $\left|u_{\hat{i}}\right| \sim|\tilde{\partial} \epsilon| \ll 1$. In particular, this is the case if the matter is dust (as viewed by the observer). The condition $\left|\alpha_{\hat{i} \mid k}^{\mid k} / H^{2}\right| \ll 1$ then also follows automatically.

\section{Light propagation}

\subsection{The redshift}

Most cosmological observations probe redshifts and distances. Let us first consider the redshift. The redshift measured by the observer is given by the change in photon energy between emission and observation, $1+z=E_{\mathrm{e}} / E_{\mathrm{o}}$. In the geometrical optics approximation, light travels on null geodesics [52] (page 93), [53], and the energy is

$$
\begin{aligned}
E & =-u_{\alpha} k^{\alpha} \\
& \simeq k^{0}[1+\mathcal{O}(\tilde{\partial} \epsilon)],
\end{aligned}
$$

where $k^{\alpha}$ is the photon momentum, tangent to a null geodesic. It is useful to split $k^{\alpha}$ as

$$
k^{\alpha}=E\left(u^{\alpha}+e^{\alpha}\right)
$$

where $u_{\alpha} e^{\alpha}=0, e_{\alpha} e^{\alpha}=1$. We define $\frac{\mathrm{d}}{\mathrm{d} \eta} \equiv\left(u^{\alpha}+e^{\alpha}\right) \partial_{\alpha}$. The component $k^{0}$ is determined by the null geodesic equation

$$
\begin{aligned}
0 & =k^{\alpha} \nabla_{\alpha} k^{0} \\
& =k^{\alpha} \partial_{\alpha} k^{0}+\Gamma_{\alpha \beta}^{0} k^{\alpha} k^{\beta} \\
& \simeq k^{\alpha} \partial_{\alpha} k^{0}+H f_{i j} k^{i} k^{j}+\mathcal{O}\left(H k^{0} k^{0} \tilde{\partial} \epsilon\right) \\
& \simeq k^{\alpha} \partial_{\alpha} k^{0}+H k^{0} k^{0}+\mathcal{O}\left(H k^{0} k^{0} \tilde{\partial} \epsilon\right),
\end{aligned}
$$

where we have on the last line used the null condition $g_{\alpha \beta} k^{\alpha} k^{\beta}=0$. From (3.1) and (3.3) we have

$$
\begin{aligned}
1+z & \simeq \exp \left(\int_{\mathrm{e}}^{\mathrm{o}} \mathrm{d} \eta[H+\mathcal{O}(H \tilde{\partial} \epsilon)]\right) \\
& \simeq\left(\frac{a_{\mathrm{e}}}{a_{\mathrm{o}}}\right)^{-1}[1+\mathcal{O}(\tilde{\partial} \epsilon)] .
\end{aligned}
$$

The redshift is to first approximation given by the inverse of the background scale factor. In other words, as long as metric perturbations are small (and the other assumptions hold), emission which is nearly isotropic at the source looks nearly isotropic to the observer. The converse is not true: near-isotropy of the redshift of the cosmic microwave background does not imply that the metric would be close to FRW [54]. 


\subsection{The distance}

While the relation between the redshift and the background scale factor remains to leading order unchanged from the FRW case, we cannot say whether changes in the redshift are small or large unless we know how the background scale factor is related to observables. More generally, the redshift is only observationally meaningful if expressed in relation to other observable quantities, such as the angular diameter distance or the age of the universe. In particular, the redshift-distance relation can change significantly, because the change in the angular diameter distance $D_{A}$ can be large ${ }^{4}$. The reason why the redshift remains close to its background value is that the photon momentum is given by a first order differential equation where first derivatives of the metric enter via the Christoffel symbols, and second derivatives do not make an appearance. In contrast, the equation for the angular diameter distance is second order. We have

$$
\frac{\mathrm{d}^{2} D_{A}}{\mathrm{~d} \lambda^{2}}=-\left[4 \pi G_{\mathrm{N}}\left(\rho+p-2 q_{\alpha} e^{\alpha}+\pi_{\alpha \beta} e^{\alpha} e^{\beta}\right) E^{2}+\tilde{\sigma}^{2}\right] D_{A},
$$

where $\frac{\mathrm{d}}{\mathrm{d} \lambda} \equiv k^{\alpha} \nabla_{\alpha}$ and $\tilde{\sigma}^{2}$ is the null shear scalar; see $[46,52,53]$ for details.

When converting the derivative with respect to the affine parameter $\lambda$ to derivative with respect to the observable redshift, we have ${ }^{5}$

$$
\begin{aligned}
\frac{\mathrm{d} D_{A}}{\mathrm{~d} \lambda} & =\partial_{z} D_{A} \frac{\mathrm{d} z}{\mathrm{~d} \lambda} \\
& =\partial_{z} D_{A} E\left(u^{\alpha} \partial_{\alpha}+e^{\alpha} \partial_{\alpha}\right) z \\
& \simeq \partial_{z} D_{A} E\left[\partial_{0} z+e^{i} \partial_{i} z+\mathcal{O}\left(\tilde{\partial} \epsilon \partial_{\alpha} z\right)\right] .
\end{aligned}
$$

Because the redshift receives corrections of order $\tilde{\partial} \epsilon$, the conversion factor (3.6) involves second derivatives of the metric. While the perturbations do not substantially change the redshift, they change the relation between the redshift and the affine parameter. This corresponds to changing the local expansion rate, shear and/or acceleration $[46,56]$.

The work [57] provides an example where metric perturbations around a matterdominated spatially flat FRW background are small, and their time derivatives and first spatial derivatives are also small, and the four-velocity perturbation is small, but the angular diameter distance is very different from the background, and is designed to exactly reproduce the best-fit $\Lambda$ CDM FRW model. The model studied in [57] is spherically symmetric. If the universe is statistically homogeneous and isotropic (and has a finite homogeneity scale) and the distribution evolves slowly, it can be

\footnotetext{
${ }^{4}$ Recall that the luminosity distance $D_{L}$ is related to the angular diameter distance via $D_{L}=$ $(1+z)^{2} D_{A}$ in a general spacetime [29], [52] (page 111), [55].

${ }^{5}$ In general, this change of variables does not make sense, because the redshift is not always monotonic along the null geodesic, so there is no function $D_{A}(z)[46,56]$.
} 
argued that the change due to the spatial derivatives in (3.6) cancels in the integrals along the null geodesic over distances longer than the homogeneity scale. In that case the distance is for typical light rays to leading order determined by the average energy density, pressure and expansion rate $[46,56]$. If the pressure can be neglected, the angular diameter distance is determined by the average expansion rate and the value of the average density today. If the average expansion rate is close to the background, the distance is expected to be close to the FRW case, in agreement with Swiss Cheese studies of light propagation [58]. The argument should be studied in more detail and made more rigorous.

An alternative to the integral approach considered here is to expand $D_{A}$ as a series in $z$ (leaving aside that in the real universe there is no function $D_{A}(z)$ ), or vice versa $[59,60]$, as recently discussed in [61]. It would seem that significant variations

in different directions in the distance are expected when second derivatives of the perturbations are large. Such an expansion is only useful for small redshifts or distances, and the cancellations for the distance are expected to occur only over large scales, so the two pictures are not in contradiction.

\section{The expansion rate}

\subsection{The local expansion rate}

In addition to the redshift and the distance, we can observe the expansion rate. Let us now consider the average expansion rate, its relation to the background scale factor $a$ and the effect of perturbations on the evolution of $a$. The volume expansion rate measured by the observer is

$$
\begin{aligned}
\theta^{(u)} & =\nabla_{\alpha} u^{\alpha} \\
& =\partial_{\alpha} u^{\alpha}+\Gamma_{\alpha \beta}^{\alpha} u^{\beta} \\
& \simeq 3 H+\partial_{i} u^{i}+\mathcal{O}(H \tilde{\partial} \epsilon)
\end{aligned}
$$

where we on the last line applied the metric (2.6). As $\partial_{i} u^{i} \sim \mathcal{O}\left(H \tilde{\partial}^{2} \epsilon\right)$, the local expansion rate can have large variations in different regions. However, the presence of large local variations does not necessarily mean that the average expansion rate would change significantly; that depends on the distribution of the fluctuations.

We assume that the observers are moving non-relativistically with respect to the averaging frame, $v \ll 1$; to simplify the bookkeeping, we assume that $v \lesssim \mathcal{O}(\tilde{\partial} \epsilon)$. When considering averages, it is useful to decompose vectors and tensors in the direction orthogonal to and directions parallel to the averaging hypersurface $\mathcal{N}$, instead of the background time and space directions. To this end, we split $n^{\alpha}$ into a vector $m^{\alpha}$ whose gradient gives (approximately) the background expansion rate $3 H$ and a vector $p^{\alpha}$ which lies along $\mathcal{N}$. We define the former by setting $m^{\alpha}=\delta^{\alpha 0}$ in 
the coordinates $(2.6)$, and the latter by

$$
p^{\alpha} \equiv n^{\alpha}+\frac{m^{\alpha}}{m^{\beta} n_{\beta}}
$$

with the components $p^{0} \simeq \mathcal{O}(\epsilon, \tilde{\partial} \epsilon \tilde{\partial} \epsilon), p^{i}=n^{i} \simeq \mathcal{O}(\tilde{\partial} \epsilon)$. With these definitions, we have

$$
\begin{aligned}
\theta^{(u)} & =g^{\alpha \beta} \nabla_{\beta} u_{\alpha} \\
& =\gamma\left(\hat{\nabla}_{\alpha} n^{\alpha}+\hat{\nabla}_{\alpha} v^{\alpha}+A_{\alpha} v^{\alpha}\right)+\gamma^{3}\left(v^{\alpha} v^{\beta} \hat{\nabla}_{\beta} v_{\alpha}+v^{\alpha} n^{\beta} \nabla_{\beta} v_{\alpha}\right) \\
& \simeq \hat{\nabla}_{\alpha} n^{\alpha}+\hat{\nabla}_{\alpha} v^{\alpha}+\mathcal{O}\left(H \tilde{\partial} \epsilon \tilde{\partial} \epsilon \tilde{\partial}^{2} \epsilon\right) \\
& =-\frac{1}{m^{\beta} n_{\beta}} \hat{\nabla}_{\alpha} m^{\alpha}+\frac{1}{\left(m^{\beta} n_{\beta}\right)^{2}} m^{\alpha} \hat{\nabla}_{\alpha}\left(m^{\gamma} n_{\gamma}\right)+\hat{\nabla}_{\alpha}\left(p^{\alpha}+v^{\alpha}\right)+\mathcal{O}\left(H \tilde{\partial} \epsilon \tilde{\partial} \epsilon \tilde{\partial}^{2} \epsilon\right) \\
& \simeq 3 H+\hat{\nabla}_{\alpha} s^{\alpha}+\mathcal{O}\left(H \tilde{\partial} \epsilon \tilde{\partial} \epsilon \tilde{\partial}^{2} \epsilon\right),
\end{aligned}
$$

where we have defined $s^{\alpha} \equiv p^{a}+v^{\alpha}$. Like $p^{\alpha}$ and $v^{\alpha}$ (but unlike $u^{i}$ ), $s^{\alpha}$ is a vector along $\mathcal{N}$. For the shear we have similarly

$$
\begin{aligned}
\sigma_{\alpha \beta}^{(u)} & =h_{\alpha(\mu}^{(u)} h_{\nu) \beta}^{(u)} \nabla^{\nu} u^{\mu}-\frac{1}{3} h_{\alpha \beta}^{(u)} \theta^{(u)} \\
& \simeq \hat{\nabla}_{\beta} s_{\alpha}-\frac{1}{3} h_{\alpha \beta} \hat{\nabla}_{\gamma} s^{\gamma}+\mathcal{O}\left(H \tilde{\partial} \epsilon \tilde{\partial}^{2} \epsilon\right) .
\end{aligned}
$$

\subsection{The average expansion rate}

As we want to average $\theta^{(u)}$ over $\mathcal{N}$, we need the relation between the background time $\bar{t}$ and the time which is constant on $\mathcal{N}$, which we denote by $t$ (note that unless $A^{\alpha}=0, t$ is not a proper time). We have

$$
\begin{aligned}
\partial_{t} \bar{t} & =n^{\alpha} \partial_{\alpha} \bar{t} \\
& =n^{0} \\
& \simeq 1+\mathcal{O}(\tilde{\partial} \epsilon \tilde{\partial} \epsilon)
\end{aligned}
$$

and $\hat{\nabla}_{\alpha} \bar{t} \sim \mathcal{O}(\tilde{\partial} \epsilon)$, so the difference between the times $\bar{t}$ and $t$ is small. Nevertheless, the difference in the volume element between the hypersurfaces of constant $\bar{t}$ and constant $t$ can be large. If the spatial coordinates differ by $\mathcal{O}\left(H^{-1} \tilde{\partial} \epsilon\right)$, the Jacobian of the coordinate transformation is $\mathcal{O}\left(\tilde{\partial}^{2} \epsilon\right)$, which is of the same order as the density perturbations. (For dust this is rather obvious: because mass is conserved, the density is inversely proportional to the volume element.) For an explicit example in a case where the hypersurface of averaging is taken to be the hypersurface of constant proper time measured by observers, see [40]. The average of the expansion rate (4.3) on $\mathcal{N}$ is

$$
\begin{aligned}
\left\langle\theta^{(u)}\right\rangle & \simeq\left\langle 3 H(\bar{t})+\hat{\nabla}_{\alpha} s^{\alpha}+\mathcal{O}\left(H \tilde{\partial} \epsilon \tilde{\partial} \epsilon \tilde{\partial}^{2} \epsilon\right)\right\rangle \\
& \simeq 3 H(t)+\left\langle\hat{\nabla}_{\alpha} s^{\alpha}\right\rangle+\mathcal{O}\left(H \tilde{\partial} \epsilon \tilde{\partial} \epsilon \tilde{\partial}^{2} \epsilon\right) \\
& \simeq 3 H(t)+\mathcal{O}\left(H \tilde{\partial} \epsilon /(H L), H \tilde{\partial} \epsilon \tilde{\partial} \epsilon \tilde{\partial}^{2} \epsilon\right)
\end{aligned}
$$


where $\left\langle\hat{\nabla}_{\alpha} s^{\alpha}\right\rangle$ reduces to a boundary term which is suppressed by $\mathcal{O}(\partial \epsilon)$ and enhanced by $1 /(H L)$, where $L^{3}$ is the proper volume of the averaging region. As long as the region is not so much smaller than the background Hubble scale that it would overcome the smallness of $s^{\alpha} \sim \mathcal{O}(\tilde{\partial} \epsilon)$, the boundary term remains subdominant. There can be large local fluctuations, but they cancel over large volumes. For this

argument it is crucial that $\hat{\nabla}_{\alpha}$ is a derivative along $\mathcal{N}$ and $s^{\alpha}$ is a vector on $\mathcal{N}$. The assumption that time derivatives of perturbations are not large is also essential; without it, all large second derivative terms would not reduce to suppressed boundary terms.

If the distribution on $\mathcal{N}$ is statistically homogeneous and isotropic and the averaging region is at least as large as the homogeneity scale, any total derivative would give a small contribution, even if the amplitude of the vector field were not small. The reason is that a total derivative corresponds to flux through the boundary, and without a preferred direction this should be equal in both directions across the boundary, up to statistical fluctuations $[22,25,46]$. Boundary terms vanish identically for periodic boundary conditions, which are used in simulations and implicitly assumed in Fourier series decomposition.

We have established that the average expansion rate is close to the background quantity $H$. The averaging hypersurface $\mathcal{N}$ has been kept general, up to the condition that the difference between the four-velocity $n^{\alpha}$ orthogonal to $\mathcal{N}$ and the observer four-velocity $u^{\alpha}$ is small. In general, different hypersurfaces of averaging give different results $[62,63]$, and relevant averages are those which give an approximate description of observable quantities. Arguments about cancellations in integrals related to the redshift and the distance indicate that these are the averages taken on the hypersurface of statistical homogeneity and isotropy $[14,22,46,56]$. However, we see that varying the choice of hypersurface does not change the leading order result as long as the difference between the two frames is non-relativistic [46]. It was argued in [46] that the observationally relevant expansion rate is $\nabla_{\alpha} n^{\alpha}$, which is related to the hypersurface of statistical homogeneity and isotropy, while we have considered $\nabla_{\alpha} u^{\alpha}$. Nevertheless, their averages are close, because the difference between the two frames is non-relativistic.

\subsection{The background expansion rate}

We have established that the redshift and the expansion rate are given in terms of the background scale factor in the same way as in FRW universes. However, this does not necessarily mean that their relation to time would be the same as in the FRW case, because the evolution of the scale factor $a$ (or equivalently the background expansion rate $H=\dot{a} / a$ ) could be different. In usual perturbation theory, equations are split up in powers of the metric perturbations, and equations at each order are assumed to be satisfied separately [50]. In particular, the evolution of background quantities is taken to be independent of the perturbations. However, this is an extra 
assumption which does not follow from the equations of motion. At linear order, the equations for background quantities are the same as in the FRW case (as long as the average of the linear perturbations vanishes). Beyond linear order, the average of the perturbations does not vanish, and when derivatives of the perturbations become large, higher order terms could have a significant impact on the average.

Let us see what happens in the present case, when second derivatives of the perturbations can be even larger than the background quantities, and we do not assume that the equations are satisfied order by order. From (2.5) and (2.7)-(2.9), we have

$$
\begin{aligned}
\rho & \simeq 3 H^{2}+3 \frac{K}{a^{2}}+2 \Psi_{\mid k}^{\mid k}+\mathcal{O}\left(\tilde{\partial} \epsilon \nabla^{2} \alpha_{\hat{i}}, \tilde{\partial} \epsilon \tilde{\partial} \epsilon \partial^{2} \epsilon, \epsilon \partial^{2} \epsilon, \bar{\partial} \Gamma \epsilon\right) \\
-p & \simeq 2 \frac{\ddot{a}}{a}+H^{2}+\frac{K}{a^{2}}+\frac{2}{3}(\Psi-\Phi)_{\mid k}^{\mid k}+\mathcal{O}\left(\tilde{\partial} \epsilon \nabla^{2} \alpha_{\hat{i}}, \tilde{\partial} \epsilon \tilde{\partial} \epsilon \partial^{2} \epsilon, \epsilon \partial^{2} \epsilon, \partial \bar{\Gamma} \epsilon\right) .
\end{aligned}
$$

Let us first average (4.7) on $\mathcal{N}$. The first two terms depend only on the background time $\bar{t}$, which is close to the time $t$, so we get simply $\left\langle 3 H(\bar{t})^{2}+3 \frac{K}{a(\bar{t})^{2}}\right\rangle \simeq$ $3 H(t)^{2}+3 \frac{K}{a(t)^{2}}+\mathcal{O}\left(H^{2} \tilde{\partial} \epsilon\right)$. For the third term we have

$$
2\left\langle\Psi_{\mid k}^{\mid k}\right\rangle \simeq 2\left\langle\hat{\nabla}_{\alpha} \hat{\nabla}^{\alpha} \Psi\right\rangle+\mathcal{O}\left(\tilde{\partial} \epsilon \tilde{\partial} \epsilon \partial^{2} \epsilon\right)
$$

This is a total derivative of a vector that has a small amplitude, so it is suppressed on the same grounds as before. The average of (4.8) is analogous, and we obtain

$$
\begin{aligned}
& \langle\rho\rangle \simeq 3 H(t)^{2}+3 \frac{K}{a(t)^{2}}+\mathcal{O}\left(H \partial \epsilon /(H L), \tilde{\partial} \epsilon \nabla^{2} \alpha_{\hat{i}}, \tilde{\partial} \epsilon \tilde{\partial} \epsilon \partial^{2} \epsilon, \epsilon \partial^{2} \epsilon, \partial \bar{\Gamma} \epsilon\right) \\
& \langle p\rangle \simeq-2 \frac{\partial_{t}^{2} a(t)}{a(t)}-H(t)^{2}-\frac{K}{a(t)^{2}}+\mathcal{O}\left(H \partial \epsilon /(H L), \tilde{\partial} \epsilon \nabla^{2} \alpha_{\hat{i}}, \tilde{\partial} \epsilon \tilde{\partial} \epsilon \partial^{2} \epsilon, \epsilon \partial^{2} \epsilon, \partial \bar{\Gamma} \epsilon\right)
\end{aligned}
$$

In other words, to leading order the evolution of $a$ is given by the FRW equations (note that here $a$ and $H$ are functions of the physical time $t$, not the background time $\bar{t}$. The average expansion rate and the redshift are therefore related to the time $t$ in the same way as the background quantities are related to $\bar{t}$, up to small corrections.

In [24], it was argued that the magnitude of the corrections to the average expansion rate cannot be resolved in usual perturbation theory once the density perturbations become non-linear. The reason was that when the expansion rate is written as a series in powers of the metric perturbation, the contribution of higher order terms is not suppressed, and the series expansion becomes useless when second derivatives of the perturbations become large. The feature of the present treatment which makes it possible to establish the amplitude of the corrections is that second derivatives of the perturbations are not treated perturbatively. The infinite series discussed in section 2.1 of [24] arises from expanding the denominator of $u_{\hat{i}}$ in (2.10) in a power series, though this is not obvious in a perturbative treatment. Here we utilise the feature 
that the average of $\partial_{i} u^{i}$ reduces (approximately) to a boundary term at any order in perturbation theory. This fact has not been recognised in previous perturbation theory studies $[7,9,24-27,37-43,61]$ (for further references and discussion, see [24]).

\section{Discussion}

\subsection{The Buchert equations}

The Buchert equations show the effect of deviations from homogeneity and isotropy on the average expansion rate in general terms $[11,12,64]$. Let us see how the above result for the average expansion rate emerges from them. We consider the expansion rate $\theta=\nabla_{\alpha} n^{\alpha}$; as noted above, the difference between the average of $\theta$ and the average of $\theta^{(u)}$ is small. The average of the expansion rate $\theta$ evolves according to the equations [46]

$$
\begin{aligned}
\partial_{t}\langle\theta\rangle+\frac{1}{3}\langle\theta\rangle^{2} \simeq & -\frac{1}{2}(\langle\rho\rangle+3\langle p\rangle)+\mathcal{Q} \\
& +\mathcal{O}\left(\tilde{\partial} \epsilon \partial^{2} \epsilon /(L H), \tilde{\partial} \epsilon \nabla^{2} \alpha_{\hat{i}}, \tilde{\partial} \epsilon \tilde{\partial} \epsilon \tilde{\partial}^{2} \epsilon \partial^{2} \epsilon\right) \\
\frac{1}{3}\langle\theta\rangle^{2} \simeq & \langle\rho\rangle-\frac{1}{2}\left\langle{ }^{(3)} R\right\rangle-\frac{1}{2} \mathcal{Q}+\mathcal{O}\left(\tilde{\partial} \epsilon \tilde{\partial} \epsilon \tilde{\partial}^{2} \epsilon \partial^{2} \epsilon\right) \\
\partial_{t}\langle\rho\rangle+\langle\theta\rangle(\langle\rho\rangle+\langle p\rangle) \simeq & -\langle\theta p\rangle+\langle\theta\rangle\langle p\rangle-\left\langle\sigma_{\alpha \beta} \pi^{\alpha \beta}\right\rangle \\
& +\mathcal{O}\left(H \nabla^{2} \alpha_{\hat{i}} /(L H), \partial \epsilon \nabla^{2} \alpha_{\hat{i}}, H^{3} \tilde{\partial} \epsilon \tilde{\partial} \epsilon \tilde{\partial}^{2} \epsilon \tilde{\partial}^{2} \epsilon\right),
\end{aligned}
$$

where we have taken into account that $A^{\alpha} \sim \mathcal{O}(\partial \epsilon)$ and that the related time dilation (i.e. deviation of $t$ from proper time) is small. (Note that $\nabla^{2} \alpha_{\hat{i}}$ makes an appearance as a boundary term, which is suppressed for large averaging volumes if we assume statistical homogeneity and isotropy, or if we assume that $\left|q_{\hat{i}}\right| \lesssim H^{2}$.) Here ${ }^{(3)} R$ is the spatial curvature scalar on $\mathcal{N}$, and the backreaction variable $\mathcal{Q}$ is

$$
\begin{aligned}
\mathcal{Q} & \equiv \frac{2}{3}\left(\left\langle\theta^{2}\right\rangle-\langle\theta\rangle^{2}\right)-\left\langle\sigma_{\alpha \beta} \sigma^{\alpha \beta}\right\rangle \\
& =\left\langle\hat{\nabla}_{\alpha} n^{\alpha} \hat{\nabla}_{\beta} n^{\beta}-\hat{\nabla}_{\beta} n_{\alpha} \hat{\nabla}^{\beta} n^{\alpha}\right\rangle-\frac{2}{3}\left\langle\hat{\nabla}_{\alpha} n^{\alpha}\right\rangle^{2} \\
& \simeq\left\langle\hat{\nabla}_{\alpha} p^{\alpha} \hat{\nabla}_{\beta} p^{\beta}-\hat{\nabla}_{\beta} p_{\alpha} \hat{\nabla}^{\beta} p^{\alpha}\right\rangle-\frac{2}{3}\left\langle\hat{\nabla}_{\alpha} p^{\alpha}\right\rangle^{2}+\mathcal{O}\left(\tilde{\partial} \epsilon \tilde{\partial}^{2} \epsilon \partial^{2} \epsilon\right) \\
& =\left\langle\hat{\nabla}_{\alpha}\left(p^{\alpha} \hat{\nabla}_{\beta} p^{\beta}-p^{\beta} \hat{\nabla}_{\beta} p^{\alpha}\right)+p^{\beta}\left[\hat{\nabla}_{\alpha}, \hat{\nabla}_{\beta}\right] p^{\alpha}\right\rangle-\frac{2}{3}\left\langle\hat{\nabla}_{\alpha} p^{\alpha}\right\rangle^{2}+\mathcal{O}\left(\tilde{\partial} \epsilon \tilde{\partial}^{2} \epsilon \partial^{2} \epsilon\right) \\
& =\left\langle\hat{\nabla}_{\alpha}\left(p^{\alpha} \hat{\nabla}_{\beta} p^{\beta}-p^{\beta} \hat{\nabla}_{\beta} p^{\alpha}\right)\right\rangle-\left\langle{ }^{(3)} R_{\alpha \beta} p^{\alpha} p^{\beta}\right\rangle-\frac{2}{3}\left\langle\hat{\nabla}_{\alpha} p^{\alpha}\right\rangle^{2}+\mathcal{O}\left(\tilde{\partial} \epsilon \tilde{\partial}^{2} \epsilon \partial^{2} \epsilon\right),
\end{aligned}
$$

where we have on the second line used the definitions of $\theta$ and $\sigma_{\alpha \beta}$ given in (2.3), and

${ }^{(3)} R_{\alpha \beta}$ is the spatial curvature tensor. (We have also used the fact that to leading order $\hat{\nabla}_{\beta} p_{\alpha}=\hat{\nabla}_{(\beta} p_{\alpha)}$.) The boundary terms are small for the same reasons as before and the spatial curvature contribution is suppressed by two powers of $p^{\alpha} \sim \mathcal{O}(\tilde{\partial} \epsilon)$. 
This structure where $\mathcal{Q}$ is almost a boundary term is close to the Newtonian case, as we discuss in section 5.3 .

The integrability condition between (5.1) and (5.2) reads

$$
\begin{aligned}
& \partial_{t}\left\langle{ }^{(3)} R\right\rangle+\frac{2}{3}\langle\theta\rangle\left\langle{ }^{(3)} R\right\rangle=-\partial_{t} \mathcal{Q}-2\langle\theta\rangle \mathcal{Q}-2\langle\theta p\rangle+2\langle\theta\rangle\langle p\rangle-2\left\langle\sigma_{\alpha \beta} \pi^{\alpha \beta}\right\rangle \\
& +\mathcal{O}\left(H \nabla^{2} \alpha_{\hat{i}} /(L H), \partial \epsilon \partial^{2} \epsilon /(L H), \partial \epsilon \nabla^{2} \alpha_{\hat{i}}, H^{3} \tilde{\partial} \epsilon \tilde{\partial} \epsilon \tilde{\partial}^{2} \epsilon \tilde{\partial}^{2} \epsilon\right)
\end{aligned}
$$

so if $\mathcal{Q}$ is small and the other terms are small (which would have to be looked at separately), the average spatial curvature evolves in the same manner as in the FRW case. The local spatial curvature scalar is

$$
\begin{aligned}
{ }^{(3)} R & =2 G_{\alpha \beta} n^{\alpha} n^{\beta}-\frac{2}{3} \theta^{2}+\sigma_{\alpha \beta} \sigma^{\alpha \beta} \\
& \simeq 6 \frac{K}{a^{2}}+4 \Psi_{\mid k}^{\mid k}-4 H \hat{\nabla}_{\alpha} p^{\alpha}-\hat{\nabla}_{\alpha}\left(p^{\alpha} \hat{\nabla}_{\beta} p^{\beta}-p^{\beta} \hat{\nabla}_{\beta} p^{\alpha}\right)+\mathcal{O}\left(\tilde{\partial} \epsilon \tilde{\partial}^{2} \epsilon \partial^{2} \epsilon\right) \\
& \simeq 6 \frac{K}{a^{2}}+4 \frac{1}{a^{2} f} \nabla^{2} \Psi-4 H \hat{\nabla}_{\alpha} p^{\alpha}-\hat{\nabla}_{\alpha}\left(p^{\alpha} \hat{\nabla}_{\beta} p^{\beta}-p^{\beta} \hat{\nabla}_{\beta} p^{\alpha}\right)+\mathcal{O}\left(\tilde{\partial} \epsilon \tilde{\partial}^{2} \epsilon \partial^{2} \epsilon\right)
\end{aligned}
$$

When the density contrast is non-linear, there are typically large local variations in the spatial curvature, like in the expansion rate. In the average, these large deviations cancel up to boundary terms, and the leading behaviour is the same as in the FRW case, $\left\langle{ }^{(3)} R\right\rangle \simeq 6 K / a(t)^{2}+\mathcal{O}\left(\tilde{\partial} \epsilon \tilde{\partial}^{2} \epsilon \partial^{2} \epsilon\right)$. If $\Psi$ is constant in time, the time-dependence of the first term in (5.6) is also $a^{-2}$, and it can be viewed as a "renormalisation" of

the background spatial curvature constant $K[26]$. In general, $\Psi$ depends on time, and such an interpretation is not valid.

\subsection{The redshift and the average expansion rate}

In a general spacetime, the redshift measured by an observer is

$$
1+z=\exp \left(\int_{\mathrm{e}}^{\mathrm{o}} \mathrm{d} \eta\left[\frac{1}{3} \theta^{(u)}+A_{\alpha}^{(u)} e^{\alpha}+\sigma_{\alpha \beta}^{(u)} e^{\alpha} e^{\beta}\right]\right) .
$$

It might appear that the change in the redshift due to perturbations would be of the order of the change in the average expansion rate. As we have seen, the latter reduces to a boundary term which, while small for sufficiently large regions, may be important for small domains. However, according to section 3.1, the change in redshift is always small under our assumptions, irrespective of the distance travelled by the light. Let us see how these facts are reconciled. We have

$$
\begin{aligned}
& \int_{\mathrm{e}}^{\mathrm{o}} \mathrm{d} \eta\left(\frac{1}{3} \theta^{(u)}+A_{\alpha}^{(u)} e^{\alpha}+\sigma_{\alpha \beta}^{(u)} e^{\alpha} e^{\beta}\right) \\
\simeq & \int_{\mathrm{e}}^{\mathrm{o}} \mathrm{d} \eta\left[H+\frac{1}{3} \hat{\nabla}_{\alpha} s^{\alpha}+u^{\beta} \nabla_{\beta} u_{\alpha} e^{\alpha}+\left(\hat{\nabla}_{\beta} s_{\alpha}-\frac{1}{3} h_{\alpha \beta} \hat{\nabla}_{\gamma} s^{\gamma}\right) e^{\alpha} e^{\beta}+\mathcal{O}\left(H \tilde{\partial} \epsilon \tilde{\partial}^{2} \epsilon\right)\right]
\end{aligned}
$$




$$
\begin{aligned}
& \simeq \ln \frac{a_{\mathrm{o}}}{a_{\mathrm{e}}}+\int_{\mathrm{e}}^{\mathrm{o}} \mathrm{d} \eta\left[\hat{\nabla}_{\beta} s_{\alpha} e^{\alpha} e^{\beta}+\mathcal{O}\left(H \tilde{\partial} \epsilon \tilde{\partial}^{2} \epsilon\right)\right] \\
& =\ln \frac{a_{\mathrm{o}}}{a_{\mathrm{e}}}+\int_{\mathrm{e}}^{\mathrm{o}} \mathrm{d} \eta\left[e^{\beta} \hat{\nabla}_{\beta}\left(s_{\alpha} e^{\alpha}\right)-s_{\alpha} e^{\beta} \hat{\nabla}_{\beta} e^{\alpha}+\mathcal{O}\left(H \tilde{\partial}_{\epsilon} \tilde{\partial}^{2} \epsilon\right)\right] \\
& =\ln \frac{a_{\mathrm{o}}}{a_{\mathrm{e}}}+\int_{\mathrm{e}}^{\mathrm{o}} \mathrm{d} \eta\left[\frac{\mathrm{d}\left(s_{\alpha} e^{\alpha}\right)}{\mathrm{d} \eta}-u^{\beta} \nabla_{\beta}\left(s_{\alpha} e^{\alpha}\right)+\mathcal{O}\left(H \tilde{\partial} \epsilon \tilde{\partial}^{2} \epsilon\right)\right] \\
& \simeq \ln \frac{a_{\mathrm{o}}}{a_{\mathrm{e}}}+\left.\right|_{\mathrm{e}} ^{\mathrm{o}} s_{\alpha} e^{\alpha}+\mathcal{O}\left(\tilde{\partial} \epsilon \tilde{\partial}^{2} \epsilon\right) \\
& \simeq \ln \frac{a_{\mathrm{o}}}{a_{\mathrm{e}}}+\mathcal{O}\left(\tilde{\partial} \epsilon \tilde{\partial}^{2} \epsilon\right) .
\end{aligned}
$$

The leading order deviation in the local expansion rate cancels with a term in the projected shear. The remaining part $e^{\alpha} e^{\beta} \hat{\nabla}_{\beta} s_{\alpha}$ is locally large, but at leading order it reduces to a total derivative in $\eta$ and thus to a small boundary term. In [56] it was noted that while the cancellation (up to a boundary term) between the expansion rate and the shear in $\mathcal{Q}$ can be understood in terms of the Newtonian limit, it is not clear whether their cancellation in the redshift could be understood in a similar manner. We now see that it follows from the smallness of $u_{\hat{i}}$ and the smallness of perturbations of the Christoffel symbols.

It is only the sum of the contributions of $\theta-3 H$ and $\sigma_{\alpha \beta}^{(u)} e^{\alpha} e^{\beta}$ which is small, not either term individually. In a statistically homogeneous and isotropic space where the distribution evolves slowly, the integral of $\sigma_{\alpha \beta}^{(u)} e^{\alpha} e^{\beta}$ alone should be strongly suppressed for typical light rays over long distances (because $\sigma_{\alpha \beta}^{(u)}$ has no preferred directions while $e^{\alpha}$ varies only slowly) $[46,56]^{6}$. It then follows that the contribution of $\theta-3 H$ is also small, in agreement with the argument that the contribution of the expansion rate is given by the spatial average (which is close to $3 H$ ) if the space is statistically homogeneous and isotropic and the distribution evolves slowly $[46,56]$.

If matter consists of discrete clumps instead of a continuous fluid, it has been argued that there could be a large effect on the redshift [65]. Given the above results, this would imply that the spacetime cannot be written in terms of a near-FRW metric and a small $u_{\hat{i}}$, or that the geometrical optics approximation is not valid [46]

\subsection{Relation to Newtonian gravity}

In Newtonian cosmology, the Raychaudhuri equation (5.1) is identical to its general relativity counterpart $[29,66]$. In contrast, the counterpart of the Hamiltonian constraint (5.2) emerges only as the first integral of the Raychaudhuri equation, whereas in general relativity it is an independent equation. This difference corresponds to the absence of spatial curvature in Newtonian gravity [29]. This is related to the fact that there are no covariant derivatives, only ordinary derivatives, which commute; as

\footnotetext{
${ }^{6}$ To be precise, the argument should be formulated in the $n^{\alpha}$ frame in terms of the decomposition (2.3) of $\nabla_{\beta} n_{\alpha}[46]$.
} 
a result, the backreaction variable $\mathcal{Q}$ contains only boundary terms [11]. When the system is isolated, i.e. boundary terms vanish, the first integral of (5.1) gives (5.2) with $\mathcal{Q}=0$ and a conserved energy term proportional to $a^{-2}$. For this reason the evolution of the scale factor in the Newtonian theory is always the same as in the FRW case, regardless of the amplitude of perturbations: in particular, accelerating expansion due to inhomogeneities is not possible ${ }^{7}$.

In contrast, in general relativity the Hamiltonian constraint (5.2) involves the average spatial curvature term, which can have non-trivial evolution [16,22,24,28,67]. However, if perturbations of the Christoffel symbols are small, the spatial structure remains close to Newtonian theory (apart from possible background curvature). Absence of spatial curvature is related to the Newtonian constraint that the magnetic component of the Weyl tensor vanishes, $H_{\alpha \beta}=0[29,31,68-70]$. In general relativity, the magnetic part of the Weyl tensor decomposed with respect to $n^{\alpha}$ is

$$
H_{\alpha \beta}=\epsilon_{\gamma \delta(\alpha} \hat{\nabla}^{\gamma} \sigma_{\beta)}^{\delta},
$$

where $\epsilon_{\alpha \beta \gamma} \equiv \eta_{\alpha \beta \gamma \delta} n^{\delta}$ is the volume element on $\mathcal{N}$, with $\eta_{\alpha \beta \gamma \delta}$ being the spacetime volume element. In the Newtonian limit, the shear can be written as $\sigma_{\alpha \beta}=\hat{\nabla}_{\alpha} \hat{\nabla}_{\beta} \phi-$ $\frac{1}{3} h_{\alpha \beta} \hat{\nabla}_{\gamma} \hat{\nabla}^{\gamma} \phi$, where $\phi$ is a scalar function identified with the gravitational potential $[29,68,69]$. We then have

$$
H_{\alpha \beta}=\epsilon_{\gamma \delta(\alpha} \hat{\nabla}^{\gamma} \hat{\nabla}^{\delta} \hat{\nabla}_{\beta)} \phi=\frac{1}{2} \epsilon_{\gamma \delta(\alpha}\left[\hat{\nabla}^{\gamma}, \hat{\nabla}^{\delta}\right] \hat{\nabla}_{\beta)} \phi=\frac{1}{2} \epsilon_{\gamma \delta(\alpha}{ }^{(3)} R_{\beta)}^{\gamma \delta}{ }_{\beta} \hat{\nabla}_{\epsilon} \phi .
$$

The three-dimensional Riemann tensor ${ }^{(3)} R_{\alpha \beta \gamma \delta}$ vanishes if and only if ${ }^{(3)} R_{\alpha \beta}$ does, because the Weyl tensor is zero in three dimensions. This relates the absence of spatial curvature and backreaction in Newtonian gravity to the lack of propagating degrees of freedom ${ }^{8}$. For the metric (2.6) and our approximation of treating metric perturbations and their first derivatives as small, $\Phi, \Psi$ and $\chi_{i j}$ and their derivatives do not contribute to $H_{\alpha \beta}$ at leading order, while second derivatives of $\alpha_{i}$ do enter and take the system far from the Newtonian behaviour, as also happens with $G_{0 i}$. (In general relativity, for an irrotational dust fluid, $H_{\alpha \beta}$ is trivially zero in usual perturbation theory at first order, implying that the theory has a linearisation instability [71]; see also [72]. We have not assumed that the matter is dust.)

In the usual post-Newtonian formalism [73] (page 86), [74] it is assumed that $\Phi, \Psi \sim \epsilon, \alpha_{i} \sim \epsilon^{3 / 2}$ and $\chi_{i j} \sim \epsilon^{2}$. If in the present context we were to similarly assume that $\alpha_{i}$ is smaller than $\Phi$ and $\Psi$ so that its second derivatives are small, the situation would be closer to the usual post-Newtonian formulation. If we start with only scalar

\footnotetext{
${ }^{7}$ In Newtonian gravity, it is also impossible to get acceleration by introducing exotic matter with negative pressure, because pressure does not gravitate.

${ }^{8}$ In general relativity, the magnetic component $H_{\alpha \beta}$ and the electric component $E_{\alpha \beta}$ have coupled evolution equations, which have wave solutions. In the Newtonian theory, $H_{\alpha \beta}$ is zero and $E_{\alpha \beta}$ does not have an evolution equation.
} 
perturbations at linear order and solve the equations of motion order by order, then at second order we have $\alpha_{\hat{i}} \sim \epsilon \tilde{\partial} \epsilon$ and $\chi_{i j} \sim \epsilon^{2}$ [75]. However, it is not clear whether this hierarchy persists once second derivatives of perturbations become large and the equations cannot be solved order by order. In the post-Newtonian formalism, each time derivative further reduces the order of magnitude by $\epsilon$, whereas spatial derivatives do not change it. In contrast, in the present approach we assume that time derivatives are (at most) of the same order of magnitude as the background, while spatial derivatives increase the order of magnitude. (The post-Newtonian formalism is constructed around Minkowski space, so the background does not involve a scale.)

It might seem promising to study backreaction in a post-Newtonian approximation. However, an essential feature of the usual post-Newtonian scheme is that the system is finite and isolated, which is not the case in cosmology. In fact, Newtonian gravity has a well-defined initial value problem only for isolated systems, periodic boundary conditions or fractal distributions with vanishing mean density $[31,35,36,76-78]$. Related to this, the non-relativistic limit of taking the speed of light to infinity is singular, so solutions of the limiting Newtonian equations are in general not limits of solutions of the relativistic equations [32,36]. Nevertheless, there has been work on post-Newtonian formulations of cosmology [36,69,79-81], all in contexts where metric perturbations are assumed to remain small. A comparison of Newtonian cosmological simulations and relativistic analytical treatment was made in [82] for a specific spherically symmetric dust configuration. Good agreement was found between the two theories in this highly symmetric case.

\subsection{Local and global backgrounds}

As long as the metric and the four-velocity remain close to FRW, there is no significant backreaction (with the caveats we have mentioned). The Christoffel symbols are given by first derivatives of the metric, and we assume that first derivatives of perturbations are small. Therefore powers of the perturbed Christoffel symbols higher than the first are negligible, and the structure remains close to linear theory. Even though variation in the Riemann tensor can be large, the geodesic equation involves only the Christoffel symbols, so the effect of curvature is locally small for light propagation and for timelike geodesic motion.

The result does not imply that in order for backreaction to be significant, there would have to be large local deviations in the Christoffel symbols. It simply means that all regions should not be close to the same global background [83]. Metric perturbations and their first spatial derivatives can still remain small with regard to a local background, which is different in different regions. With reference to a global background, the metric perturbations or their first spatial derivatives in some regions would then be large.

For example, consider a stabilised region with a constant non-zero density, such

as a dark matter halo, in an expanding spatially flat dust background. From (4.7) it 
follows that $\nabla^{2} \Psi$ has a decaying part proportional to $a^{-1}$ (corresponding to the falling background expansion rate) and a growing a part proportional to $a^{2}$ (corresponding to the constant density). In terms of the background coordinate time $\bar{t}$, the perturbation $\Psi$ will become larger than unity as the universe expands. This does not mean that there would be locally strong gravitational effects: metric perturbations remain small (away from regions of high mass concentration) if the metric is expanded around Minkowski space in the local region. It is only the difference between the global background and the appropriate local background which is diverging. Of course, the evolution of the metric perturbations should be determined in detail from the equations of motion, and this argument only shows that the metric perturbation $\Psi$ of a stabilised region grows initially like $a^{2}$ if the perturbations and their derivatives are initially small. There is a complication in that it would be more appropriate to consider the proper time measured by observers (or at least the time $t$ which is constant on $\mathcal{N}$ ) and not the unphysical background time $\bar{t}$. Even if the two times are near each other, the difference in the corresponding spatial derivatives is of order unity once second derivatives of the metric become large. It is therefore not straightforward to extract the proper time dependence of $\Psi$ from the background time dependence of $\nabla^{2} \Psi$.

Possibly ordinary perturbation could be used as a null test: by calculating quantities such as the variance of the expansion rate $[84]^{9}$ in perturbation theory and comparing to observations we might try to rule out the assumption that perturbation theory holds. However, to obtain predictions, it is usually not enough to know that the amplitude of the metric perturbations is small, their evolution has to be known as well.

Straightforward perturbation theory with regard to the metric is not suited to the case when metric perturbations are not small around a global background, but are instead small only with regard to local backgrounds which are different in different regions. A full analytical treatment is unfeasible, but it might be possible to obtain a simplified system of equations which could be treated via statistical methods $[22,23]$ or numerical simulations. One possibility could be to use the covariant formulation of the evolution and constraint equations [29,47-49], which deals directly with physical degrees of freedom, so that the problem can be discussed without assumptions about perturbativity of the metric around some background. However, it is not clear whether there is a tractable approximation which would include the cosmologically relevant degrees of freedom.

Let us emphasise how the present treatment differs from previous work [7,9,24, 37-43] (see [24] for more references and discussion). Most previous studies apply either usual or post-Newtonian perturbation theory, where all quantities are expanded

\footnotetext{
${ }^{9}$ The study [84] was mistakenly criticised in $[22,24]$ for misestimating the magnitude of the boundary term. However, the analysis is still constrained by the applicability of second order perturbation theory.
} 
in a series of either the metric perturbation or peculiar velocity, and solved order by order. However, when second derivatives of metric perturbations become large in cosmology, this procedure is inconsistent, because second order quantities can be larger than first order quantities, $\tilde{\partial}^{2} \epsilon \tilde{\partial}^{2} \epsilon \gg \epsilon$. In contrast, we have not assumed that second derivatives of metric perturbations are small or split the quantities order by order. Therefore our result applies beyond usual perturbation theory. Also, we have defined the averaging hypersurface using physical criteria, taken the volume element into account, and correctly identified the local expansion rate measured by the observer, unlike in some previous work. We have also studied the observable redshift and angular diameter distance directly.

Recently, an interesting approach has been introduced to tackle the backreaction problem without having to assume that second derivatives are small [44]. The idea is to consider a family of metrics $g_{\alpha \beta}(\lambda)$ which depend on some small parameter $\lambda$. The background is identified as the metric $g_{\alpha \beta}(0)$, and perturbation theory is developed in terms of $\lambda$. A novelty of the formalism is that space and time derivatives of the metric are not assumed to be analytic in $\lambda$, and they have a well-defined limit as $\lambda \rightarrow 0$ only when smeared over a local region of the background spacetime. It is then shown (with some other assumptions) that if the metric is close to the background, the background satisfies the Einstein equation with only an additional effective radiation term, and corrections due to perturbations are small. The formalism is interesting in that the notion of a homogeneity scale is incorporated into the analysis in a natural manner, and the calculations are mathematically rigorous. However, the connection to the real universe is somewhat unclear. The physical interpretation of the parameter $\lambda$ and the adopted scaling of various quantities with $\lambda$ is not obvious. In the present case, we use standard general relativity, and there are no extra assumptions to be made. (However, in [44], it is not assumed that the first derivatives of metric perturbations would be small.) Note that it is important to consider correctly defined observables, as smoothing and calculating observables do not in general commute: in [44], the redshift and the angular diameter distance are not considered. The cautionary example [57] shows that small metric perturbations and small peculiar velocities do not guarantee that changes to the angular diameter distance are small. Also, as noted in [83], the local smoothing considered in [44] is a different procedure from averaging over large scales. (The smoothing in [44] is done with respect to the background space, not the physical space.) To the extent one can make a comparison, the results of [44] and the present work do not appear to be in disagreement. Both studies share the weakness that their starting point is that the spacetime is close to FRW, an assumption which should be carefully looked at.

\section{Conclusion}

It has been claimed that the effect of non-linear structures on the average expansion 
rate and on light propagation is small in the real universe, because the metric remains close to FRW at all times. The argument has two parts: that the metric remains close to FRW, and that this implies that the change in the average expansion rate and light propagation is small. We have considered the second part of the argument. The relevant quantities depend not only on the metric, but also on its first and second derivatives. Second derivatives of metric perturbations have variations of order unity after structures become non-linear, so smallness of the metric perturbations alone is not a sufficient condition for backreaction to be small. We have made the assumptions that time derivatives and first spatial derivatives of metric perturbations are small and the perturbation of the observer four-velocity is small. (The last assumption implies certain conditions on second derivatives of the metric and/or the matter content.) It then follows that the redshift is to leading order given by the background scale factor. We further assume that the difference between the observer four-velocity and the four-velocity which defines the hypersurface of averaging is small. The difference between the average expansion rate and the background expansion rate then reduces to a boundary term which is small as long as the averaging domain is not much smaller than the Hubble scale, and the background expansion rate evolves in the same manner as in the FRW case. This can be understood from the fact that perturbations of the Christoffel symbols remain small, so the structure is close to Newtonian cosmology, where backreaction reduces to a boundary term [11].

However, even with these assumptions, perturbations can have a large effect on the angular diameter distance, as demonstrated in [57]. It has been argued that if the space is statistically homogeneous and isotropic and the distribution evolves slowly, then the distance is determined by the average expansion rate, and the change in the distance is small, too $[46,56]$. The issue should be studied in more detail.

The assumptions needed for the proof show that smallness of metric perturbations and their time derivatives and first spatial derivatives is not sufficient for the effect on the redshift and the average expansion rate to be small. The assumption about the smallness of the deviation of the observer four-velocity $u^{\alpha}$ from the background is crucial. In general, the deviation of $u^{\alpha}$ from the background is a gauge-dependent quantity which cannot be straightforwardly identified as the deviation from the physical mean flow determined from observations. Nevertheless, if we assume that the observer moves along a geodesic (which is the realistic case in the late universe), the smallness of the deviation of $u^{\alpha}$ follows from the assumptions about metric perturbations and their derivatives. It would be useful to have a definition of the peculiar velocity that would be valid in a general cosmological spacetime [51] and that would correspond to the observational use of the term, so that the observed smallness of this quantity could be used as an input.

If backreaction is significant, its effect cannot be expressed in terms of a changed FRW background nor small perturbations around a FRW universe $[14,16,22,24,46$, 56]. Rather, perturbations can remain small only with respect to a local region, and 
if we insist on a global background metric instead of a patchwork of backgrounds, then metric perturbations or perturbations of the four-velocity have to be large. The important issue is the behaviour of physical quantities, not in which form the metric or four-velocity can be written. In the real universe, fluctuations of the Riemann tensor and the expansion rate are not small: local variations of the expansion rate are of the same order as the measured deviation of the average expansion rate from the matter-dominated FRW value. The question is whether the distribution of local values is such that variations cancel when considering the average expansion rate, the redshift and the distance. Further focusing on perturbativity, perhaps in the context of a patchwork of regions, might be a useful way towards reliably quantifying backreaction.

\section{Acknowledgments}

I thank Carl Bender, Stephen Green, Hans Mühlen, Dominik Schwarz, Robert Wald and Clifford Will for useful discussions and Washington University in St Louis, the University of Chicago, LAPTH and the Pettersson Institute for Theoretical Physicists for their hospitality.

\section{References}

[1] M. Vonlanthen, S. Räsänen and R. Durrer, Model-independent cosmological constraints from the CMB, JCAP08(2010)023 [arXiv:1003.0810 [astro-ph.CO]]

[2] N. Jackson, The Hubble Constant, Living Reviews in Relativity 10 (2007) 4 [0709.3924 [astro-ph]] G.A. Tammann, A. Sandage and B. Reindl, The expansion field: The value of $H_{0}$, Astron. ${ }^{3}$ Astrophys. 15 (2008) 289 [arXiv:0806.3018 [astro-ph]]

A.G. Riess et al., A Redetermination of the Hubble Constant with the Hubble Space Telescope from a Differential Distance Ladder, Astrophys. J. 699 (2009) 539 [arXiv:0905.0695 [astro-ph.CO]] A.G. Riess et al., A 3\% Solution: Determination of the Hubble Constant with the Hubble Space Telescope and Wide Field Camera 3, Astrophys. J. 730 (2011) 119 [arXiv:1103.2976 [astro-ph.CO]]

[3] L.M. Krauss and B. Chaboyer, Age Estimates of Globular Clusters in the Milky Way: Constraints on Cosmology, Science 299 (2003) 65

[4] P.J.E. Peebles, Probing General Relativity on the Scales of Cosmology [arXiv:astro-ph/0410284]

[5] T. Buchert, On average properties of inhomogeneous cosmologies, 2000, Proc. 9th JGRG conference, ed. Y. Eriguchi et al., p 306 [arXiv:gr-qc/0001056]

[6] T. Tatekawa, M. Suda, K.-i. Maeda and H. Kubotani, Inhomogeneities in Newtonian Cosmology and its Backreaction to the Evolution of the Universe [arXiv:astro-ph/0109501]

[7] C. Wetterich, Can Structure Formation Influence the Cosmological Evolution?, Phys. Rev. D67 (2003) 043513 [arXiv:astro-ph/0111166]

[8] D.J. Schwarz, Accelerated expansion without dark energy [arXiv:astro-ph/0209584] 
[9] S. Räsänen, Dark energy from backreaction, JCAP02(2004)003 [arXiv:astro-ph/0311257]

S. Räsänen, Backreaction of linear perturbations and dark energy [arXiv:astro-ph/0407317]

[10] G.F.R. Ellis, Relativistic cosmology: its nature, aims and problems, 1984, The invited papers of the 10th international conference on general relativity and gravitation, D. Reidel

Publishing Co., Dordrecht, p 215

G.F.R. Ellis and W. Stoeger, The 'fitting problem' in cosmology, Class. Quant. Grav. 4 (1987) 1697

[11] T. Buchert and J. Ehlers, Averaging inhomogeneous Newtonian cosmologies, Astron. 8 Astrophys. 320 (1997) 1 [arXiv:astro-ph/9510056]

[12] T. Buchert, On average properties of inhomogeneous fluids in general relativity I: dust cosmologies, Gen. Rel. Grav. 32 (2000) 105 [arXiv:gr-qc/9906015]

[13] G.F.R. Ellis and T. Buchert, The universe seen at different scales, Phys. Lett. A347 (2005) 38 [arXiv:gr-qc/0506106]

[14] S. Räsänen, Accelerated expansion from structure formation, JCAP11(2006)003 [arXiv:astro-ph/0607626]

[15] T. Buchert, Dark Energy from Structure - A Status Report, Gen. Rel. Grav. 40 (2008) 467 [arXiv:0707.2153 [gr-qc]]

[16] S. Räsänen, Backreaction as an alternative to dark energy and modified gravity [arXiv:1012.0784 [astro-ph.CO]]

S. Räsänen, Backreaction: directions of progress, Class. Quant. Grav. 28 (2011) 164008 [arXiv:1102.0408 [astro-ph.CO]]

[17] C.H. Chuang, J.A. Gu and W.Y. Hwang, Inhomogeneity-Induced Cosmic Acceleration in a Dust Universe, Class. Quant. Grav. 25 (2008) 175001 [arXiv:astro-ph/0512651]

[18] A. Paranjape and T.P. Singh, The Possibility of Cosmic Acceleration via Spatial Averaging in Lemaître-Tolman-Bondi Models, Class. Quant. Grav. 23 (2006) 6955 [arXiv:astro-ph/0605195]

[19] T. Kai, H. Kozaki, K.-i. Nakao, Y. Nambu and C.M. Yoo, Can inhomogeneities accelerate the cosmic volume expansion?, Prog. Theor. Phys. 117 (2007) 229 [arXiv:gr-qc/0605120]

[20] S. Räsänen, Cosmological acceleration from structure formation, Int. J. Mod. Phys. D15 (2006) 2141 [arXiv:astro-ph/0605632]

[21] A. Paranjape, The Averaging Problem in Cosmology [arXiv:0906.3165 [astro-ph.CO]]

[22] S. Räsänen, Evaluating backreaction with the peak model of structure formation, JCAP04(2008)026 [arXiv:0801.2692 [astro-ph]]

[23] S. Räsänen, The effect of structure formation on the expansion of the universe, Int. J. Mod. Phys. D17 (2008) 2543 [arXiv:0805.2670 [astro-ph]]

S. Räsänen, Structure formation as an alternative to dark energy and modified gravity [arXiv:0811.2364 [astro-ph]]

[24] S. Räsänen, Applicability of the linearly perturbed FRW metric and Newtonian cosmology, Phys. Rev. D81 (2010) 103512 [arXiv:1002.4779 [astro-ph.CO]]

[25] A. Notari, Late time failure of Friedmann equation, Mod. Phys. Lett. A21 (2006) 2997 [arXiv:astro-ph/0503715]

[26] E.W. Kolb, S. Matarrese and A. Riotto, On cosmic acceleration without dark energy, New J. Phys. 8 (2006) 322 [arXiv:astro-ph/0506534]

[27] E.W. Kolb, S. Matarrese and A. Riotto, Comments on Backreaction and Cosmic Acceleration [arXiv:astro-ph/0511073]

[28] T. Buchert and M. Carfora, On the curvature of the present-day Universe, Class. Quant. Grav. 25 (2008) 195001 [arXiv:0803.1401 [gr-qc]]

[29] G.F.R. Ellis, Relativistic Cosmology, 1971, General Relativity and Cosmology, ed. R.K. Sachs, Academic Press Inc., London, p 104, Reprinted in Gen. Rel. Grav. 41 (2009) 581

[30] J. Ehlers, The Newtonian Limit of General Relativity, 1991, Classical mechanics and relativity: relationship and consistency, ed. G. Ferrarese, Bibliopolis, Naples, p 95 
[31] G.F.R. Ellis and P.K.S. Dunsby, Newtonian evolution of the Weyl tensor, Astrophys. J. 479 (1997) 97 [arXiv:astro-ph/9410001]

[32] J.M.M. Senovilla, C.F. Sopuerta and P. Szekeres, Theorems on shear-free perfect fluids with their Newtonian analogues, Gen. Rel. Grav. 30 (1998) 389 [arXiv:gr-qc/9702035]

[33] H. van Elst and G.F.R. Ellis, Quasi-Newtonian dust cosmologies, Class. Quant. Grav. 15 (1998) 3545 [arXiv:gr-qc/9805087]

[34] J. Ehlers, The Newtonian Limit of General Relativity, 1998, Understanding Physics, ed. A.K. Richter, Copernicus, p 1

[35] J. Ehlers, Some Developments in Newtonian Cosmology, 1999, On Einstein's Path, ed. A. Harvey, Springer-Verlag, p 189

[36] P. Szekeres and T. Rainsford, Post-Newtonian Cosmology, Gen. Rel. Grav. 32 (2000) 479 [arXiv:gr-qc/9903056]

T. Rainsford, Newtonian and Post-Newtonian Approximations of the $k=0$

Friedmann-Robertson-Walker cosmology, Gen. Rel. Grav. 32 (2000) 719

[arXiv:gr-qc/9907094]

P. Szekeres, Newtonian and Post-Newtonian Limits of Relativistic Cosmology, Gen. Rel.

Grav. 32 (2000) 1025

T. Rainsford, Anisotropic Homogeneous Cosmologies in the Post-Newtonian Approximation, Gen. Rel. Grav. 33 (2001) 1047 [arXiv:gr-qc/0007061]

[37] T. Futamase, Approximation Scheme for Constructing a Clumpy Universe in General Relativity, Phys. Rev. Lett. 61 (1988) 2175

T. Futamase, An approximation scheme for constructing inhomogeneous universes in general relativity, Mon. Not. Roy. Astron. Soc. 237 (1989) 187

S. Bildhauer and T. Futamase, The age problem in inhomogeneous universes, Gen. Rel. Grav. 23 (1991) 1251

T. Futamase, A New Description for a Realistic Inhomogeneous Universe in General Relativity, Prog. Theor. Phys. 86 (1991) 389

T. Futamase, Averaging of a locally inhomogeneous realistic universe, Phys. Rev. D53 (1996) 681

[38] U. Seljak and L. Hui, Global Expansion in an Inhomogeneous Universe, 1996, The proceedings of Clusters, Lensing and the Future of the Universe, ASP Conference Series vol 88, p 267

L. Hui and U. Seljak, Light propagation in a clumpy universe, 1996, Astrophysical applications of gravitational lensing: proceedings of the 173rd Symposium of the International Astronomical Union, ed. C.S. Kochanek and J.N. Hewitt, Kluwer Academic Publishers, Dordrecht, p 89

[39] H. Russ, M.H. Soffel, M. Kasai and G. Börner, Age of the universe: Influence of the inhomogeneities on the global expansion-factor, Phys. Rev. D56 (1997) 2044 [arXiv:astro-ph/9612218]

[40] E.W. Kolb, S. Matarrese, A. Notari and A. Riotto, The effect of inhomogeneities on the expansion rate of the universe, Phys. Rev. D71 (2005) 023524 [arXiv:hep-ph/0409038]

[41] E.R. Siegel and J.N. Fry, Effects of Inhomogeneities on Cosmic Expansion, Astrophys. J. 628 (2005) L1 [arXiv:astro-ph/0504421]

[42] A. Ishibashi and R.M. Wald, Can the acceleration of our universe be explained by the effects of inhomogeneities?, Class. Quant. Grav. 23 (2006) 235 [arXiv:gr-qc/0509108]

[43] D. Baumann, A. Nicolis, L. Senatore and M. Zaldarriaga, Cosmological Non-Linearities as an Effective Fluid [arXiv:1004.2488 [astro-ph.CO]]

[44] S.R. Green and R.M. Wald, A new framework for analyzing the effects of small scale inhomogeneities in cosmology, Phys. Rev. D83 (2011) 084020 [arXiv:1011.4920 [gr-qc]]

[45] Y.B. Zel'dovich, Observations in a universe homogeneous in the mean, Soviet Astronomy 8 (1964) 13 
[46] S. Räsänen, Light propagation in statistically homogeneous and isotropic universes with general matter content, JCAP03(2010)018 [arXiv:0912.3370 [astro-ph.CO]]

[47] J. Ehlers, Contributions to the relativistic mechanics of continuous media, Abh. Akad. Wiss. Lit. Mainz. Nat. Kl. 11 (1961) 792 (in German) Reprinted in Gen. Rel. Grav. 25 (1993) 1225

[48] G.F.R. Ellis and H. van Elst, Cosmological models (Cargèse lectures 1998), NATO Adv. Study Inst. Ser. C. Math. Phys. Sci. 541 (1999) 1 [arXiv:gr-qc/9812046]

[49] C.G. Tsagas, A. Challinor and R. Maartens, Relativistic cosmology and large-scale structure, Phys. Rept. 465 (2008) 61 [arXiv:0705.4397 [astro-ph]]

[50] M. Bruni, S. Matarrese, S. Mollerach and S. Sonego, Perturbations of spacetime: Gauge transformations and gauge invariance at second order and beyond, Class. Quant. Grav. 14 (1997) 2585 [arXiv:gr-qc/9609040]

[51] G.F.R. Ellis, H. van Elst and R. Maartens, General relativistic analysis of peculiar velocities, Class. Quant. Grav. 18 (2001) 5115 [arXiv:gr-qc/0105083]

G.F.R. Ellis and C.G. Tsagas, A relativistic approach to nonlinear peculiar velocities and the Zeldovich approximation, Phys. Rev. D66 (2002) 124015 [arXiv:astro-ph/0209143]

[52] P. Schneider, J. Ehlers and E.E. Falco, Gravitational Lenses, 1992 Springer-Verlag, New York

[53] M. Sasaki, Cosmological Gravitational Lens Equation: Its Validity And Limitation, Prog. Theor. Phys. 90 (1993) 753

[54] S. Räsänen, On the relation between the isotropy of the CMB and the geometry of the universe, Phys. Rev. D79 (2009) 123522 [arXiv:0903.3013 [astro-ph.CO]]

[55] I.M.H. Etherington, On the definition of distance in general relativity, Philosophical Magazine 15 (1933) 761 Reprinted in Gen. Rel. Grav. 39 (2007) 1055

[56] S. Räsänen, Light propagation in statistically homogeneous and isotropic dust universes, JCAP02(2009)011 [arXiv:0812.2872 [astro-ph]]

[57] K. Enqvist, M. Mattsson and G. Rigopoulos, Supernovae data and perturbative deviation from homogeneity, JCAP09(2009)022 [arXiv:0907.4003 [astro-ph.CO]]

[58] N. Brouzakis, N. Tetradis and E. Tzavara, The Effect of Large-Scale Inhomogeneities on the Luminosity Distance JCAP02(2007)013 [astro-ph/0612179]

N. Brouzakis, N. Tetradis N and E. Tzavara, Light Propagation and Large-Scale Inhomogeneities, JCAP04(2008)008 [astro-ph/0703586]

K. Bolejko, The Szekeres Swiss Cheese model and the CMB observations, Gen. Rel. Grav. 41 (2009) 1737 [arXiv:0804.1846 [astro-ph]]

R.A. Vanderveld, É.É. Flanagan and I. Wasserman, Luminosity distance in 'Swiss cheese' cosmology with randomized voids: I. Single void size, Phys. Rev. D78 (2008) 083511 [arXiv:0808.1080 [astro-ph]]

W. Valkenburg, Swiss Cheese and a Cheesy CMB JCAP06(2009)010 [arXiv:0902.4698 [astro-ph.CO]]

V. Kostov, Average luminosity distance in inhomogeneous universes, JCAP04(2010)001 [arXiv:0910.2611 [astro-ph.CO]]

S.J. Szybka, On light propagation in Swiss-Cheese cosmologies [arXiv:1012.5239 [astro-ph.CO]]

[59] J. Kristian and R.H. Sachs, Observations In Cosmology, Astrophys. J. 143 (1966) 379

[60] C.A. Clarkson, On the Observational Characteristics of Inhomogeneous Cosmologies: Undermining the Cosmological Principle [arXiv:astro-ph/0008089]

[61] C. Clarkson and O. Umeh, Is backreaction really small within concordance cosmology?, Class. Quant. Grav. 28 (2011) 164010 [arXiv:1105.1886 [astro-ph.CO]]

[62] G. Geshnizjani and R. Brandenberger, Back Reaction And Local Cosmological Expansion Rate, Phys. Rev. D66 (2002) 123507 [arXiv:gr-qc/0204074]

G. Geshnizjani and R. Brandenberger, Back Reaction Of Perturbations In Two Scalar Field Inflationary Models, JCAP04(2005)006 [arXiv:hep-th/0310265] 
[63] S. Räsänen, Backreaction in the Lemaître-Tolman-Bondi model, JCAP11(2004)010 [arXiv:gr-qc/0408097]

[64] T. Buchert, On average properties of inhomogeneous fluids in general relativity II: perfect fluid cosmologies, Gen. Rel. Grav. 33 (2001) 1381 [arXiv:gr-qc/0102049]

[65] T. Clifton and P.G. Ferreira, Archipelagian Cosmology: Dynamics and Observables in a Universe with Discretized Matter Content, Phys. Rev. D80 (2009) 103503 (errata: Phys. Rev. D84 (2011) 109902(E)) [arXiv:0907.4109 [astro-ph.CO]] T. Clifton and P.G. Ferreira, Errors in Estimating $\Omega_{\Lambda}$ due to the Fluid Approximation, JCAP10(2009)26 [arXiv:0908.4488 [astro-ph.CO]]

[66] G.F.R. Ellis, The evolution of inhomogeneities in expanding Newtonian cosmologies, Mon. Not. Roy. Astron. Soc. 243 (1990) 509

[67] S. Räsänen, Backreaction and spatial curvature in dust universes, Class. Quant. Grav. 23 (2006) 1823 [arXiv:astro-ph/0504005]

[68] L. Kofman and D. Pogosyan, Dynamics of gravitational instability is nonlocal, Astrophys. J. 442 (1995) 30

[69] S. Matarrese and D. Terranova, Post-Newtonian Cosmological Dynamics in Lagrangian Coordinates, Mon. Not. Roy. Astron. Soc. 283 (1996) 400 [arXiv:astro-ph/9511093]

[70] J. Ehlers and T. Buchert, On the Newtonian Limit of the Weyl Tensor, Gen. Rel. Grav. 41 (2009) 2153 [arXiv:0907.2645 [gr-qc]]

[71] H. van Elst, C. Uggla, W.M. Lesame, G.F.R. Ellis and R. Maartens, Integrability of Irrotational Silent Cosmological Models, Class. Quant. Grav. 14 (1997) 1151 [arXiv:gr-qc/9611002] C.F. Sopuerta, New study of silent universes, Phys. Rev. D55 (1997) 5936

[72] P.D. D'Eath, On the Existence of Perturbed Robertson-Walker Universes, Ann. Phys. 98 (1976) 237

[73] C.M. Will, Theory And Experiment In Gravitational Physics, 1981 Cambridge of University Press, Cambridge

[74] T. Futamase and B.F. Schutz, Newtonian and post-Newtonian approximations are asymptotic to general relativity, Phys. Rev. D28 (1983) 2363

B.F. Schutz, The Newtonian limit, Lecture Notes in Physics, 205 (1984) 367

T. Futamase and B.F. Schutz, Gravitational radiation and the validity of the far-zone quadrupole formula in the Newtonian limit of general relativity, Phys. Rev. D32 (1985) 2557

[75] S. Matarrese, S. Mollerach and M. Bruni, Second-order perturbations of the Einstein-de Sitter universe, Phys. Rev. D58 (1998) 043504 [arXiv:astro-ph/9707278]

[76] J. Ehlers and T. Buchert, Newtonian cosmology in Lagrangian formulation: Foundations and perturbation theory, Gen. Rel. Grav. 29 (1997) 733 [arXiv:astro-ph/9609036]

[77] J.D. Norton, The Cosmological Woes of Newtonian Gravitation Theory, The Expanding Worlds of General Relativity (Einstein Studies, volume 7), eds. H Goenner et al., p 271

[78] A. Gabrielli, M. Joyce, B. Marcos and F. Sicard, A dynamical classification of the range of pair interactions, J. Stat. Phys 141 (2010) 970 [arXiv:1003.5680v1 [cond-mat.stat-mech]]

[79] K. Tomita, Post-Newtonian Equations Of Motion In An Expanding Universe, Prog. Theor. Phys. 79 (1988) 258

K. Tomita, Post-Newtonian equations of motion in flat cosmological models, Prog. Theor. Phys. 85 (1991) 1041

M. Shibata, H. Asada, Post-Newtonian equations of motion in the flat universe, Prog.

Theor. Phys. 94 (1995) $11 \%$

T. Futamase, An approximation scheme for constructing inhomogeneous universes in general relativity, Mon. Not. Roy. Astron. Soc. 237 (1989) 187

[80] S. Matarrese, O. Pantano and D. Saez, A Relativistic approach to gravitational instability in the expanding Universe: Second order Lagrangian solutions, Mon. Not. Roy. Astron. Soc. 271 (1994) 513 [arXiv:astro-ph/9403032] 
S. Matarrese and D. Terranova, Lagrangian dynamics of collisionless matter, [arXiv:astro-ph/9601163]

C. Carbone and S. Matarrese, A Unified treatment of cosmological perturbations from super-horizon to small scales, Phys. Rev. D71 (2005) 043508 [arXiv:astro-ph/0407611] E. Villa, S. Matarrese and D. Maino, Post-Newtonian cosmological dynamics of plane-parallel perturbations and back-reaction, [arXiv:1105.1975 [astro-ph.CO]]

[81] J.-c. Hwang, H. Noh and D. Puetzfeld, Cosmological nonlinear hydrodynamics with post-Newtonian corrections, JCAP03(2008)010 [arXiv:astro-ph/0507085]

D. Puetzfeld, Beyond linearized cosmology, AIP Conf. Proc. 805 (2005) 483 [arXiv:astro-ph/0509398]

J.-c. Hwang and H. Noh, Newtonian versus relativistic nonlinear cosmology, Gen. Rel. Grav. 38 (2006) 703 [arXiv:astro-ph/0512636]

[82] D. Alonso, J. García-Bellido, T. Haugbølle and J. Vicente, Large scale structure simulations of inhomogeneous LTB void models, Phys. Rev. D82 (2010) 123530 [arXiv:1010.3453 [astro-ph.CO]].

[83] G.F.R. Ellis, Inhomogeneity effects in Cosmology [arXiv:1103.2335 [astro-ph.CO]]

[84] N. Li and D.J. Schwarz, Scale dependence of cosmological backreaction, Phys. Rev. D78 (2008) 083531 [arXiv:0710.5073 [astro-ph]]

N. Li, M. Seikel and D.J. Schwarz, Is dark energy an effect of averaging?, Fortsch. Phys. 56 (2008) 465 [arXiv:0801.3420 [astro-ph]]

M. Seikel and D.J. Schwarz, Probing Backreaction Effects with Supernova Data

[arXiv:0912.2308 [astro-ph.CO]] 